\title{
No-Break de 3kVA para aplicações residenciais com interface para painéis solares e controle microprocessado
}

\section{$3 \mathrm{kVA}$ UPS for residential application with interface for solar panels and microprocessor control}

\author{
André Luiz Batista Ferreira ${ }^{1}$; Carlos Henrique Gonçalves Treviso²; Luis Carlos \\ Mathias ${ }^{3}$; Marcus Vinicius Maia Rodrigues ${ }^{4}$; Willian Ricardo Bispo Murbak Nunes ${ }^{5}$
}

\section{Resumo}

Este artigo traz um projeto completo de um No-Break de 3kVA de saída, para aplicações residenciais, sendo uma importante contribuição para o meio acadêmico e comercial. Permite ainda a utilização de painéis solares, podendo tornar a carga em questão menos dependente da rede elétrica, além da utilização da energia limpa. Para tanto foi implementado um protótipo em campo, com banco de baterias de 150V, utilizando como controle geral um Digital Signal Controller (DSC) da família DSP56F800E da Freescale ${ }^{\mathrm{TM}}$.

Palavras-chave: Conversor, Boost, Inversor, No-Break, Painel solar, DSC, Controle em malha fechada.

\begin{abstract}
This paper presents a complete design of a UPS 3kVA for residential applications and it is an important contribution to the academic and commercial environment. It also allows the use of solar panels, can make the load in question less dependent on the grid, and the use of clean energy. To this end we implemented a prototype in the field with battery bank of $150 \mathrm{~V}$, using as general control a digital signal controller (DSC) family of DSP56F800E Freescale ${ }^{\mathrm{TM}}$.

Key words: Converter, Boost, Inverter, UPS, Solar panel, DSC, Closed loop control.
\end{abstract}

1 Docente do Departamento de Engenharia Elétrica da Universidade Estadual de Londrina - DEEL-UEL; albf@uel.br

2 Docente do Departamento de Engenharia Elétrica da Universidade Estadual de Londrina - DEEL-UEL; treviso@uel.br

3 Aluno de pós-graduação do Departamento de Engenharia Elétrica, Universidade Estadual de Londrina

4 Aluno de pós-graduação do Departamento de Engenharia Elétrica, Universidade Estadual de Londrina

5 Aluno de pós-graduação do Departamento de Engenharia Elétrica, Universidade Estadual de Londrina 


\section{Introdução}

Atualmente o consumo de energia elétrica mundial vem aumentando de forma progressiva. A maior parte desta energia é proveniente da queima de combustíveis fósseis. Como não são fontes renováveis, o uso de energia torna-se cada vez mais racional, promovendo a busca por novos meios de geração energética (FRAIDENRAICH, 2002), (COSTA, 2000).

Além disso, em nosso país, o atendimento de energia elétrica em determinadas regiões é escasso. Segundo o Sinopse do Censo Demográfico de 2010 do Instituto Brasileiro de Geografia e Estatística (IBGE, 2010), mais de 700 mil domicílios permanentes não são atendidos por nenhum tipo de geração de energia elétrica, e agravando a situação, quase $30 \%$ dos domicílios em aglomerados subnormais (favelas e comunidades, por exemplo) não dispõe desse serviço público com confiabilidade e segurança, já que seu fornecimento não é regularizado. (banco de dados IBGE, 2010).

Estes fatores certamente contribuem de maneira negativa para a sociedade brasileira, alimentando a desigualdade no Brasil.

Acrescido a essa escassez, para as localidades onde existe o fornecimento regular de energia, tem-se ainda o problema da descontinuidade do fornecimento de energia elétrica pelas concessionárias, acarretando prejuízos algumas vezes irreparáveis.

Em vista dessas considerações, a elaboração de um equipamento que consiga resolver os problemas expostos acima, que possa tornar o sistema elétrico mais confiável para o usuário final é de grande importância.

Neste sentido, este artigo traz um No-Break implementado em bancada. Neste No-Break que também foi construído em protótipo, pode-se acoplar a um sistema fotovoltaico tornando-o um gerador de energia. Como o interesse é atender tanto academicamente, quanto comercialmente do ponto de vista residencial e industrial, concluiu-se que a potência de 3kVA de saída (carga) para o No-Break seria razoável. Para tal potência, foram utilizadas 10 baterias em série formando um barramento de tensão de 150V (SOLTER, 2002), (SILVEIRA JUNIOR, SOUZA, 2004). Esse banco de baterias com $100 \%$ de carga tem uma projeção de autonomia de 25 minutos para a potência máxima. $\mathrm{O}$ sistema ainda dispõe de um controle digital, o que eleva sua confiabilidade e estabilidade, abrindo novas opções de configuração de funcionamento (SOUZA JUNIOR, PAIXÃO, 2005).

O equipamento, classificado segundo a norma brasileira NBR 15014/2003 (que define a nomenclatura das topologias de UPS no Brasil) como Standby, funciona por histerese, ou seja, através de dois níveis de carga do banco de baterias, onde será utilizada a energia proveniente da rede ou do banco de baterias, conforme a condição do funcionamento. Por exemplo, se um painel solar estiver acoplado, a energia provida será a do banco de baterias, enquanto sua carga armazenada estiver dentro da faixa que varia de $100 \%$ a $70 \%$. Ao atingir $70 \%$, a energia provida será da concessionária, mantendo assim um limite mínimo de 70\% de carga nas baterias do No-Break para uma eventual descontinuidade no fornecimento de energia elétrica da concessionária.

No intervalo de recarga do No-Break, o painel solar fornece energia para o banco de baterias. Quanto maior o número de painéis solares, menor será a necessidade do uso da energia da rede elétrica, pois menor será o tempo para recarregar as baterias, sendo que esta histerese é controlada utilizando um controle digital, baseado em DSC (Digital Signal Controller).

Visando o amplo mercado externo (principalmente na região do Mercosul e na América Latina) o No-Break desenvolvido também deve respeitar as normas internacionais, a saber, a IEC 62040-3, que classifica os No-breaks ou UPS (do termo em inglês Uninterruptible Power Supply) 
frente a fenômenos de QEE (Queda de Energia Elétrica). De acordo com esta norma, o protótipo desenvolvido será classificado como: VFD (do termo Voltage and Frequency Dependent) já que a tensão de saída pode variar com variações na tensão e na frequência de entrada quando está operando no modo rede; SX (forma de onda senoidal no modo rede e quase-quadrada no modo bateria), pois quando o equipamento opera no modo rede é a rede elétrica que alimentará diretamente a carga; e $\mathbf{2 2 2}$ (onde o tempo para a comutação da rede para a bateria e a estabilização da alimentação da carga está entre $1 \mathrm{~ms}$ e $10 \mathrm{~ms})$. Assim, a classificação completa é VFD SX 222 (SOLTER, 2002).

Para ilustrar o conceito do equipamento, a Figura 1 traz o esquema do No-Break operando em Standby em forma de diagrama de blocos, enquanto que nos apêndices, são apresentados o diagrama de blocos detalhado que representa cada um dos circuitos internos do sistema (Apêndice 1), o circuito de controle desenvolvido com DSC (Apêndice 2) e o circuito completo de potência e controle do conversor Boost (Apêndice 3).

Figura 1 - Diagrama de blocos representando o funcionamento básico do No-Break.

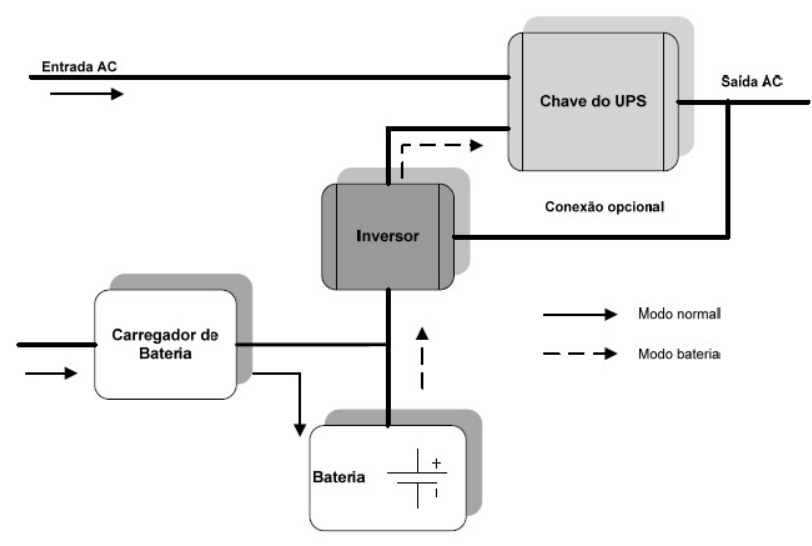

Fonte: Dados do Autor

\section{Circuitos do No-Break}

De forma a facilitar a compreensão do circuito como um todo, o No-Break foi dividido em blocos, conforme mostrado no Apêndice 1, sendo apresentado neste item o circuito simplificado e o funcionamento de cada um dos blocos.

\section{Circuito do Controle Geral (DSC)}

Para o controle geral do No-Break, foi utilizado um DSC do modelo MC56F8006 da família DSP56F800E da Freescale ${ }^{\mathrm{TM}}$ (SOUZA JUNIOR, PAIXÃO, 2005), (SILVEIRA JUNIOR, SOUZA, 2004), (FREESCALE ${ }^{\mathrm{TM}}$, 1996). Este circuito integrado possui núcleo híbrido, deste modo, o projeto se beneficia das vantagens do Processamento Digital de Sinais (PDS) e dos diversos periféricos de um microcontrolador como por exemplo o conversor Analógico para Digital (AD).

Conforme o circuito de controle desenvolvido com DSC (Apêndice 2), este controlador recebe sinais condicionados provenientes dos diversos circuitos sensores do sistema do No-Break. Os sinais das duas teclas de interface com o usuário são do tipo discreto (digital), sendo cada um condicionado por meio de um resistor de pull-up e de um capacitor para minimizar os efeitos de debounce. Já os sinais do detector de luminosidade, de monitoramento da tensão do banco de baterias, e do circuito de detecção da falta da rede elétrica são do tipo contínuo (analógico). O circuito de condicionamento dos sinais do sensor de luminosidade e da tensão no banco de baterias utilizam de divisores resistivos.

Já o condicionamento do sinal de entrada de cada uma das fases da rede é composto por um divisor resistivo (que ajusta e atenua a tensão), por um amplificador seguidor de tensão e por um filtro passa baixas RC (Resistor-Capacitor) série antes de conectado à uma entrada do conversor $\mathrm{AD}$ do DSC.

Como atuadores discretos que compõem o sistema temos os circuitos: dos três diodos emissores de luz (LED) de estado, do alarme sonoro (buzzer), do sinal de sincronismo e de shutdown 
para os demais conversores, da habilitação do funcionamento do carregador do banco de baterias, dos três pulsos de chaveamento da ponte inversora, dos dois circuitos de acionamento dos contatores de comutação entre rede elétrica e inversor.

A Figura 2 mostra a fotografia da ferramenta de desenvolvimento da Freescale ${ }^{\mathrm{TM}}$ para o DSC MC56F8006 enquanto que a Figura 3 mostra a fotografia do circuito de controle implementado.

Figura 2 - Ferramenta de desenvolvimento do DSC.

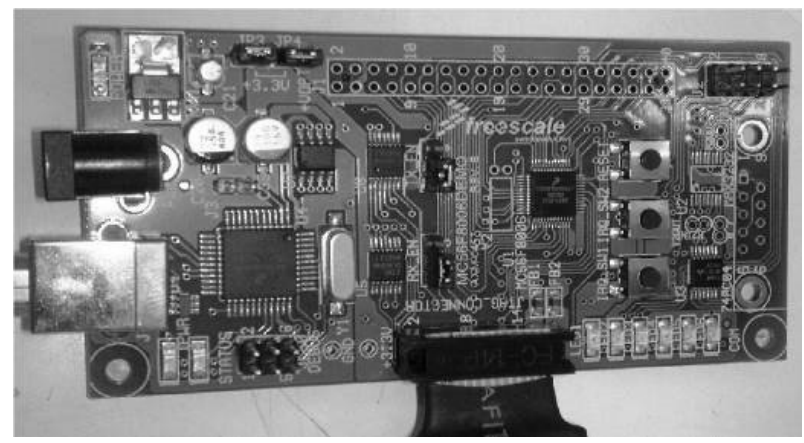

Fonte: Freescale ${ }^{\mathrm{TM}}$

Figura 3 - Circuito de controle desenvolvido com DSC.

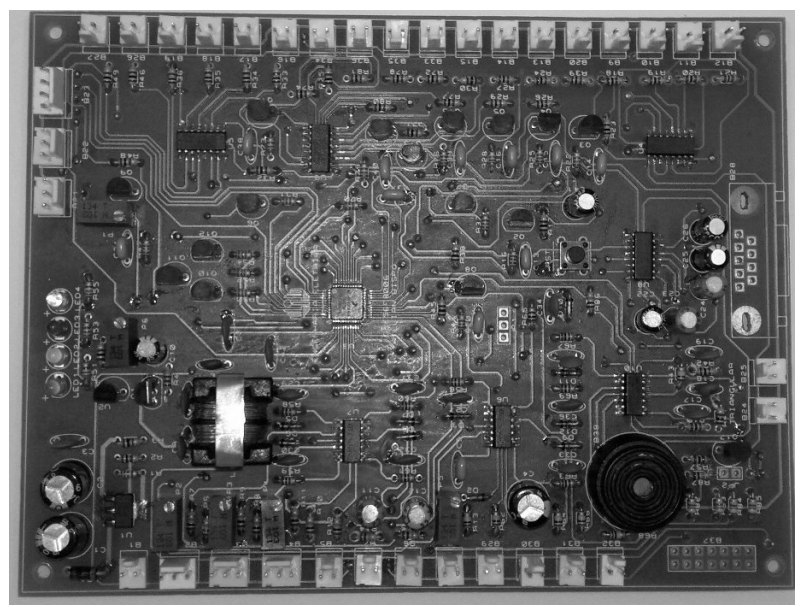

Fonte: Autor
O DSC estabelece o modo de funcionamento por uma lógica estruturada embasada numa Máquina de Estados Finitos (GILL, 1962). Assim, a partir do monitoramento dos sinais de entrada, o controle geral atua nos demais blocos do sistema, decidindo se o sistema funciona em modo de histerese ou não conforme descrito na seção introdutória deste trabalho. Com o intuito de atender à especificação também descrita na seção introdutória deste trabalho quanto ao tempo de estabilização da comutação entre rede elétrica e inversor, a detecção da presença da tensão da rede foi realizada por meio da comparação dos sinais de tensão das três fases amostradas e ressincronizadas com uma tabela de uma senóide padrão armazenada na memória flash do DSC. Assim, foi estabelecido uma margem de tolerância para a detecção da falta de rede. Se detectado elevada diferença na comparação em qualquer uma das fases, o sistema detecta falha na rede e comuta instantaneamente os consumidores para o inversor. Quando reestabilizada a rede elétrica o sistema aguarda $5 \mathrm{~s}$ para comutar do inversor para a rede enquanto a bateria não possuir mais $70 \%$ da carga e o painel fotovoltaico estiver fornecendo energia.

Além disso, ele realiza o sincronismo de todos os conversores que constituem o No-Break através de um pulso de 500ns. Gera também um sinal de shutdown quando houver necessidade de desativá-los. O DSC também é responsável por gerar os pulsos defasados em 120 graus das chaves comutadoras do inversor.

O controle geral também promove uma interface homem-máquina através de uma tela de LCD e comunicação EIA-232 com o computador. Ambos fornecem informações sobre o estado de funcionamento do No-Break. Para expansões futuras no circuito de controle foram previstos: quatro entradas analógicas de propósito geral, uma saída digital para a partida de um gerador e dois sinais auxiliares de comutação. 


\section{Conversor DC/DC}

A topologia escolhida para realizar a conversão DC/DC foi a Boost, que eleva o nível de tensão do banco de baterias para o inversor. A escolha dessa topologia deve-se ao fato de que do ponto de vista da bateria, o circuito do conversor é como uma fonte de corrente constante, o que implica em uma menor geração de ruídos na malha de terra, o que seria prejudicial considerando o uso de um controle digital (MELLO, 2011), (RASHID, 1999).

O esquema básico do conversor desenvolvido encontra-se na Figura 4.

Figura 4 - Esquema simplificado do conversor Boost.

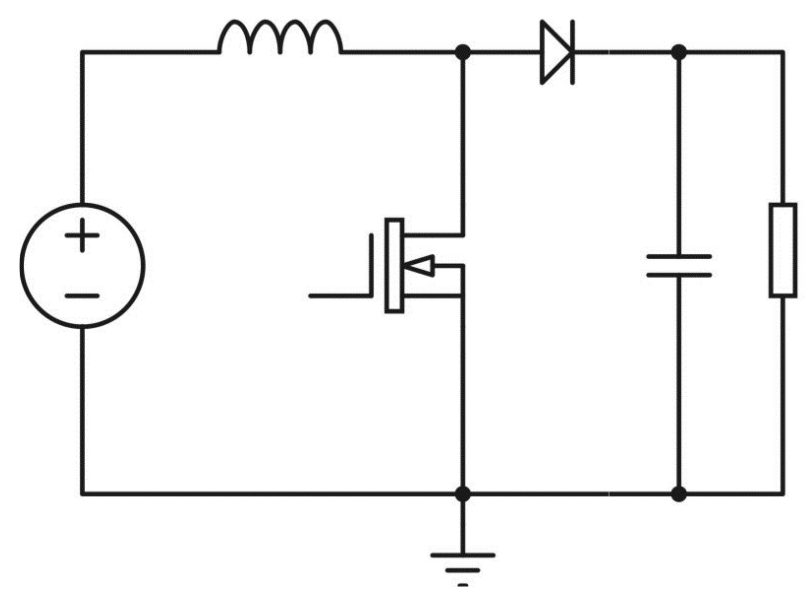

Fonte: MELLO, 2011

As especificações de projeto para o conversor DC/DC constam na Tabela 1.

\begin{tabular}{ll}
\hline Características & Valor \\
\hline Tensão máxima de entrada & $150 \mathrm{~V}$ \\
Tensão mínima de entrada & $100 \mathrm{~V}$ \\
Tensão de saída & $180 \mathrm{~V}$ \\
Frequência de operação & $50 \mathrm{kHz}$ \\
Corrente de saída & $17 \mathrm{~A}$ \\
Corrente de saída mínima & $1,7 \mathrm{~A}$ \\
Razão cíclica máxima & 0,444 \\
Potência de saída & $3060 \mathrm{~W}$ \\
\hline
\end{tabular}

Fonte: Dados do Autor
Para o circuito de controle do conversor (Figura 3) optou-se por utilizar um controle analógico com o CI SG 3525, devido a sua fácil implementação e para concentrar o controle geral do sistema NoBreak no DSC.

Desta forma, os pulsos de controle para o Boost são gerados na forma de Modulação por Largura de Pulso (PWM) através do CI SG 3525, em malha fechada, com realimentação de tensão e corrente que, amostrados ao CI, tornam-se possíveis o controle da corrente e da tensão de saída.

O controle ainda dispõe de proteção contra sobre-temperatura com acionamento de coolers quando necessário, e também uma entrada para sincronismo proveniente do controle digital com DSC, que pode sincronizar todos os pulsos de disparo que ocorrem no No-Break, diminuindo consideravelmente o ruído inerente ao sistema. Este pulso possui a duração de $500 \mathrm{~ns}$. O controle ainda dispõe de um pulso de shutdown, proveniente do DSC que é capaz de encerrar o funcionamento de todos os blocos do No-Break, incluindo o conversor Boost.

As Figuras 5 e 6 mostram as fotografias dos protótipos de potência e de controle do conversor Boost desenvolvido.

Figura 5 - Fotografia do conversor Boost desenvolvido.

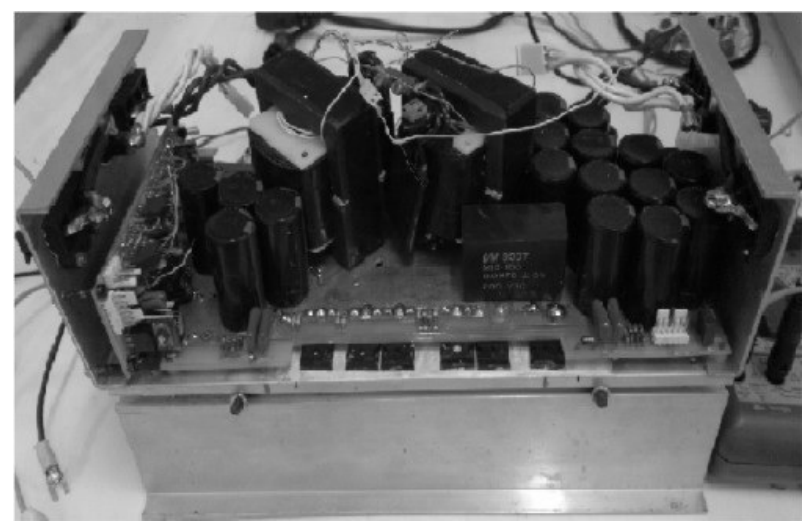

Fonte: Autor 
Figura 6 - Fotografia do controle implementado para o Boost.

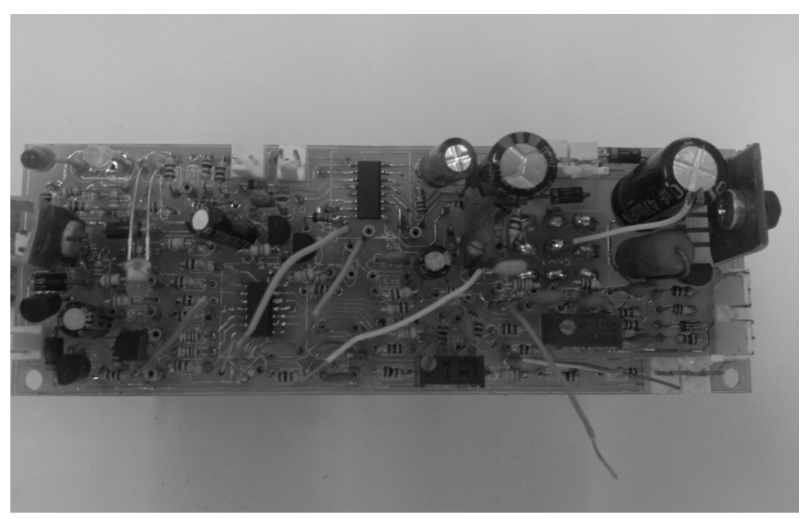

Fonte: Autor
Inversor $D C / A C$

Para obter a conversão de um sinal DC para um sinal AC, foi utilizado um inversor de fonte de tensão (VSI) monofásico com a topologia ponte-completa (RASHID, 1999), (MOHAN, UNDELAND, ROBBINS, 2003).

A forma de onda escolhida para a saída é a quasequadrada, também conhecida comercialmente como pseudo-senoidal (apresenta um melhor custobenefício para o projeto) e ajustada em $115 \mathrm{~V}_{\mathrm{RMS}} \mathrm{e}$ $60 \mathrm{~Hz}$, com modulação PWM, tendo como entrada o conversor Boost (AHMED, LEE, NAKAOKA, 2007).

O circuito simplificado do inversor está na Fig. 7, enquanto que as especificações do circuito implementado constam na Tabela 2.

Figura 7 - Esquema simplificado do Inversor de tensão.

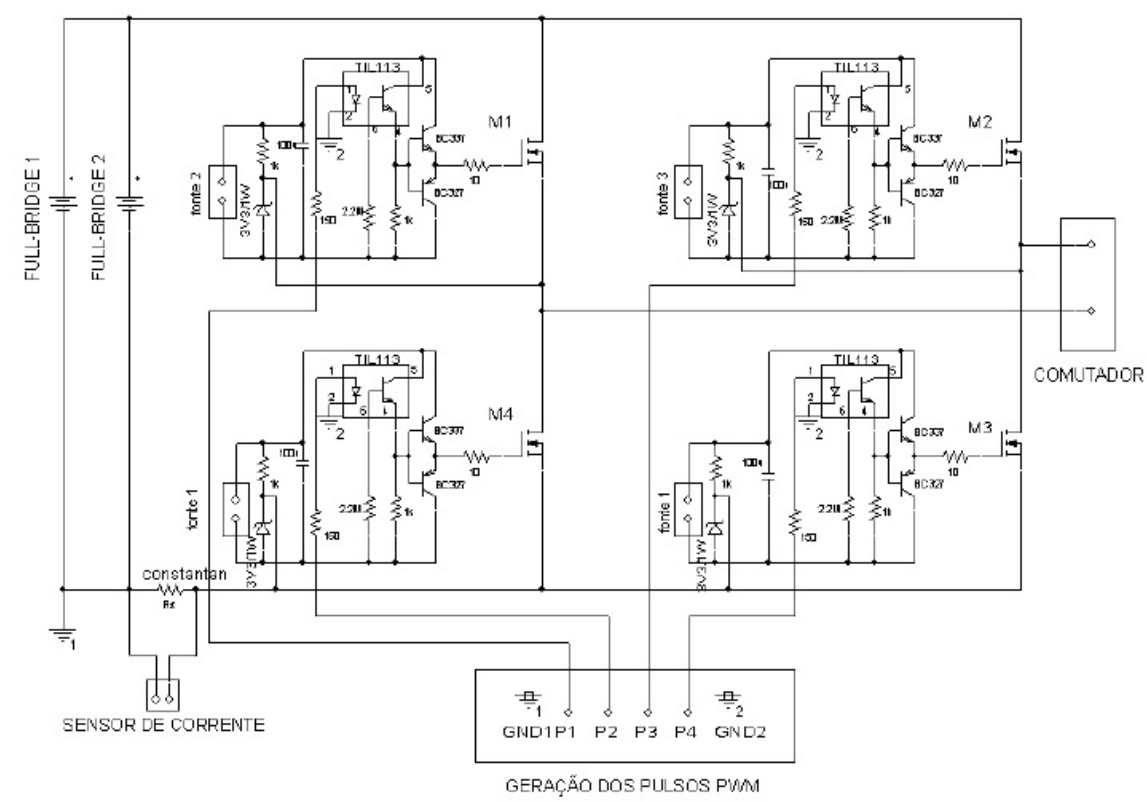

Fonte: Dados do Autor 
Tabela 2 - Especificações para o inversor de tensão.

\begin{tabular}{ll}
\hline Características & Valor \\
\hline Tensão de entrada & $180 \mathrm{~V}$ \\
Corrente de entrada & $17 \mathrm{~A}$ \\
Tensão de saída & $115 \mathrm{Vac}$ \\
Frequência de saída & $60 \mathrm{~Hz}$ \\
Corrente de saída & $26 \mathrm{~A}$ \\
Razão cíclica & 0,5 \\
Potência de entrada & $3060 \mathrm{VA}$ \\
Potência de saída & $3000 \mathrm{VA}$ \\
\hline
\end{tabular}

Fonte: Dados do Autor

Quando existe a comutação para o inversor, todas as fases da carga conectada ao No-Break estarão sob a tensão fase do inversor e neutro, ou seja, serão curto-circuitadas, tornando a saída para a carga somente monofásica.

O controle do inversor de tensão utiliza o DSC para gerar os pulsos de comando para cada uma de suas chaves e para mantê-lo sincronizado com os outros blocos. Sua malha fechada (realimentação de tensão) depende de um circuito analógico que faz uso de uma onda triangular em $120 \mathrm{~Hz}$ produzida pelo DSC.

O controle do inversor também possui proteção contra sobre-temperatura, cortando os pulsos e desligando o sistema e soft-start para amenizar o impacto sobre a carga de saída no momento que o inversor é acionado.

A fotografia do inversor montado está na Figura 8.
Figura 8 - Fotografia do inversor.

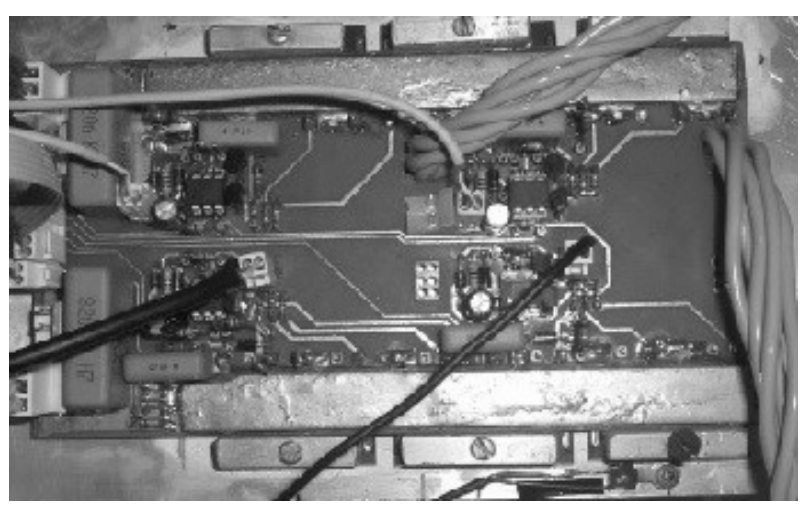

Fonte: Autor

\section{Comutador}

O bloco do comutador realiza a transferência de função de fornecimento energético da rede elétrica para o No-Break na ocasião de falta de energia, e vice-versa, quando houver a normalização da rede elétrica (AHMED, 2000), (SAMESINA, REZENDE, PEREIRA, 2006).

Essa função é realizada através de contatores trifásicos inter-travados capazes de suportar uma corrente de até 50 A. Para que ocorra a comutação, o controle geral com DSC deve detectar a falta de uma ou mais fases e enviar o comando para a realização da comutação.

Quando o painel solar é conectado ao controle do comutador, é desativado o circuito carregador, sendo utilizada a energia proveniente do sistema fotovoltaico para recarregar o banco de baterias durante o dia. No momento em que o DSC detecta que a energia das baterias está abaixo de $70 \%$ e se a rede está em condições normais, é novamente acionada a comutação com os contatores para a rede.

Se a rede elétrica não é restabelecida no intervalo de descarga das baterias, o No-Break continua fornecendo energia até o limite mínimo de carga do banco, que ao ser atingido, desliga o equipamento.

Durante a noite, o sistema é habilitado somente como No-Break, mesmo com o painel solar 
acoplado, o que implica que não haverá operação dos dois níveis de carga da bateria (histerese).

Portanto, quanto maior a potência de conversão dos painéis solares e maior a capacidade de armazenamento do banco de baterias, menos dependente torna-se o sistema da energia proveniente da rede elétrica.

\section{Carregador}

A função do carregador é, como já sugere o nome, recarregar o banco de baterias do No-Break, porém, somente na ausência do painel solar e durante a noite, utilizando energia da rede, enquanto a carga está sendo alimentada normalmente com a rede elétrica.

A topologia empregada foi a Full-Bridge, projetado com um retificador em ponte completa na entrada.

As especificações para o carregador constam na Tabela 3, onde foi considerado como tensão de entrada a tensão de saída do bloco retificador.

Tabela 3 - Especificações para o carregador.

\begin{tabular}{ll}
\hline Características & Valor \\
\hline Tensão máxima de entrada & $350 \mathrm{~V}$ \\
Tensão mínima de entrada & $260 \mathrm{~V}$ \\
Tensão de saída & $56 \mathrm{~V}$ \\
Frequência de operação & $50 \mathrm{kHz}$ \\
Corrente de saída & $3 \mathrm{~A}$ \\
Corrente de saída mínima & $0,3 \mathrm{~A}$ \\
Razão cíclica máxima & 0,45 \\
\hline
\end{tabular}

Fonte: Dados do Autor

De forma semelhante ao conversor Boost, o controle do carregador é analógico e utiliza o CI SG 3525 para gerar os pulsos de comando para os drivers das 4 chaves do conversor. O controle geral fornece os pulsos de sincronismo e de shutdown. O carregador é acionado pelo DSC através do pulso Controle Carregador, sendo este pulso determinado pelo modo de operação do sistema geral (modo histerese ou modo No-Break). A Figura 9 mostra a fotografia do carregador juntamente com seu controle.

Figura 9 - Fotografia do carregador e de seu controle.

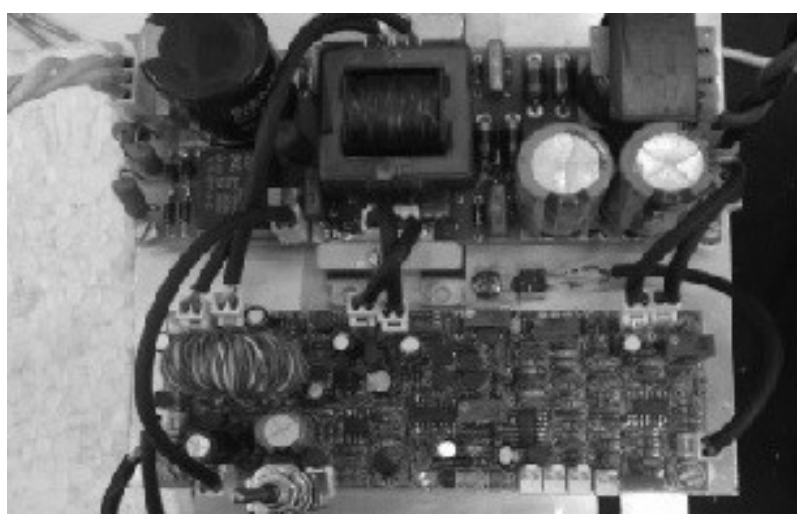

Fonte: Autor

\section{Conversor Fly-Back}

O conversor Fly-Back é o encarregado de alimentar os contatores de $48 \mathrm{~V}$ e a tensão estável de $12 \mathrm{~V}$ de todos os circuitos do No-Break, tendo como entrada o banco de baterias. Em conjunto com o circuito do Fly-Black também está o circuito acionador dos contatores.

O controle do conversor é feito de forma analógica com o CI SG3525 e recebe do controle geral com DSC os pulsos de sincronismo, de shutdown e de acionamento dos contatores.

Seu circuito simplificado consta na Figura 10. 
Figura 10 - Esquema simplificado do Fly-Back.

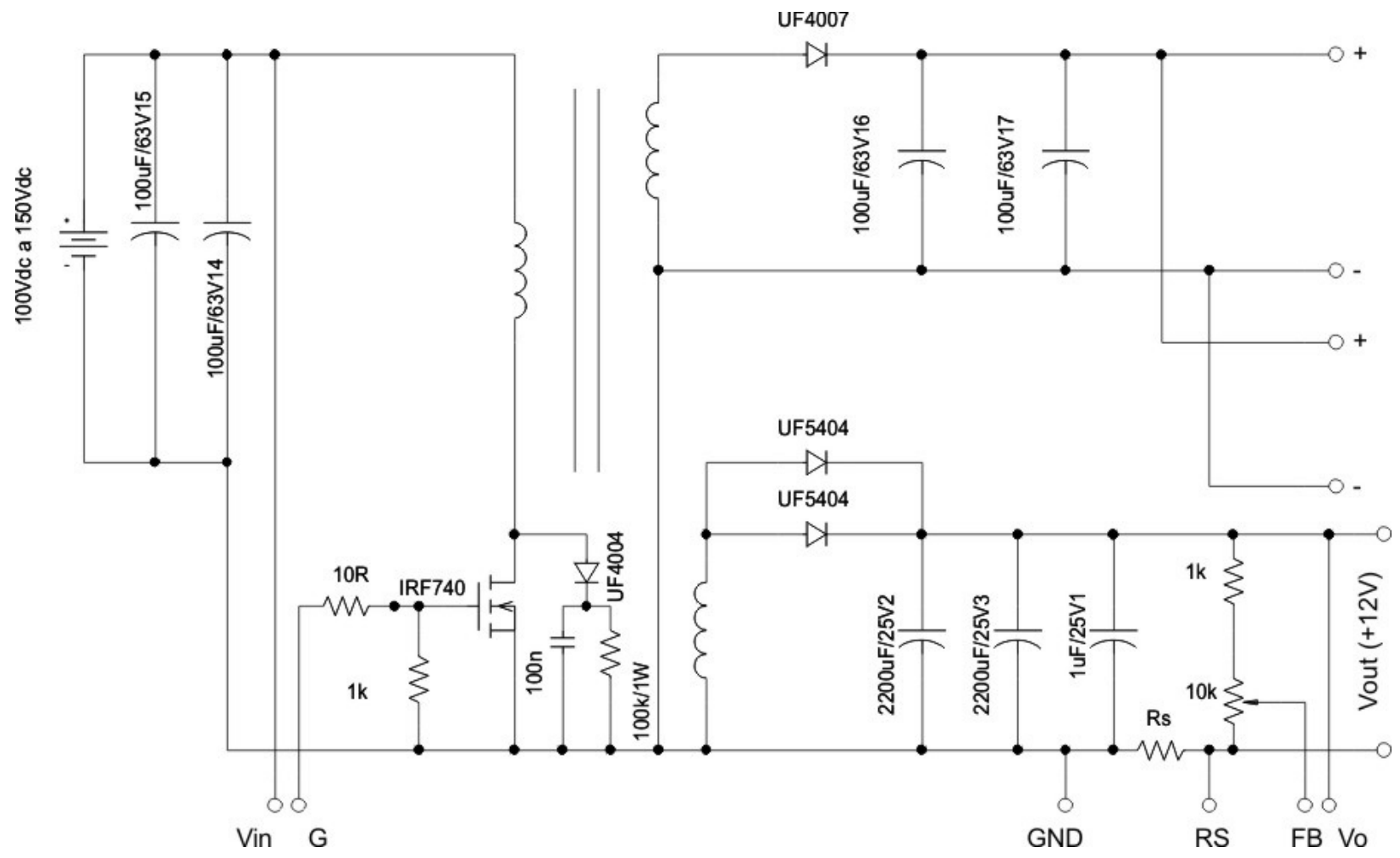

Fonte: Dados do Autor

As especificações para o Fly-Back estão relacionadas na Tabela 4.

Tabela 4 - Especificações para o Fly-Back.

\begin{tabular}{lll}
\hline Características & Saída 1 & Saída 2 \\
\hline Tensão máxima de entrada & $150 \mathrm{~V}$ & \\
Tensão mínima de entrada & $100 \mathrm{~V}$ & \\
Tensão de saída & $48 \mathrm{~V}$ & $12 \mathrm{~V}$ \\
Frequência de operação & $50 \mathrm{kHz}$ & \\
Corrente de saída & $0,3 \mathrm{~A}$ & $2 \mathrm{~A}$ \\
Corrente de saída mínima & $0,1 \mathrm{~A}$ & $0,2 \mathrm{~A}$ \\
Razão cíclica máxima & 0,50 & 0,5 \\
\hline
\end{tabular}

Fonte: Dados do Autor

$\mathrm{O}$ circuito de controle para o Fly-Back é idêntico ao que foi utilizado no conversor Boost (Figura 6).

\section{Resultados experimentais}

Para a realização dos testes do No-Break, foram empregados alicate amperímetro e voltímetro TRUE RMS, além de um osciloscópio digital para coletar as formas de onda. Foram realizados testes com cargas resistivas e indutivas, visando aproximar o experimento a situações reais de uso.

Com o auxilio do osciloscópio, foram coletadas as formas de onda para a tensão e para a corrente para cargas lineares e não lineares.

A saída do sistema de No-Break está representada nas Figuras 11 e 12, sendo que a Figura 11 mostra as formas de onda de tensão e corrente para carga linear e a Figura 12, para carga não linear. Em ambos os resultados, foram desenvolvidos a potência plena na saída do No-Break. 
Figura 11 - Formas de onda de tensão $(\mathrm{CH} 1)$ e corrente $(\mathrm{CH} 2)$ para carga puramente resistiva em potência plena (conjunto de 12 reostatos de $1 \mathrm{~kW}$ cada em paralelo formando um equivalente de $2,6 \Omega$ ).

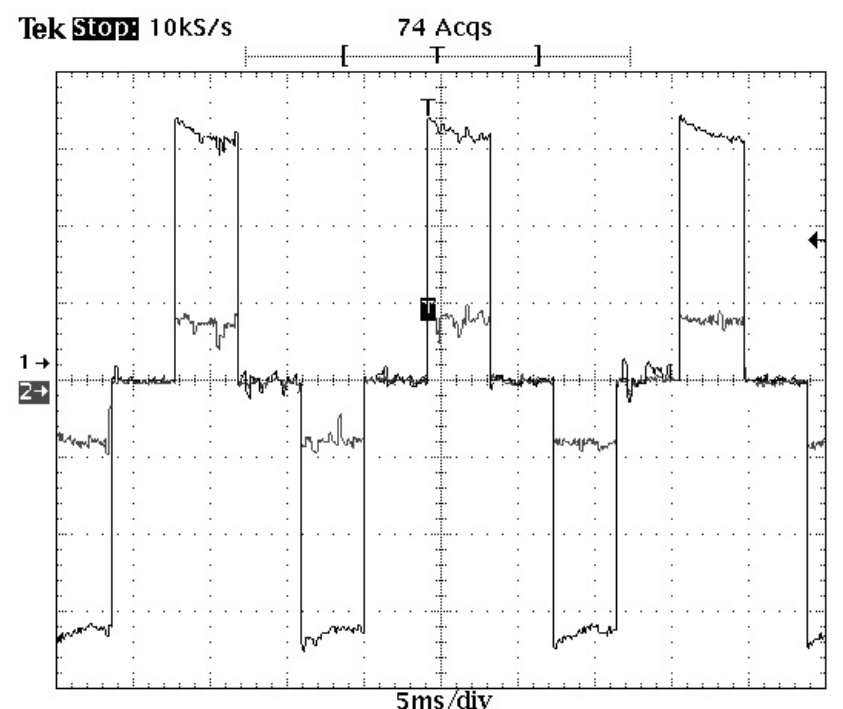

Fonte: Autor

Figura 12 - Formas de onda de tensão (CH1) e corrente $(\mathrm{CH} 2)$ para carga não linear formada por diodos, capacitores e resistores em potência plena.

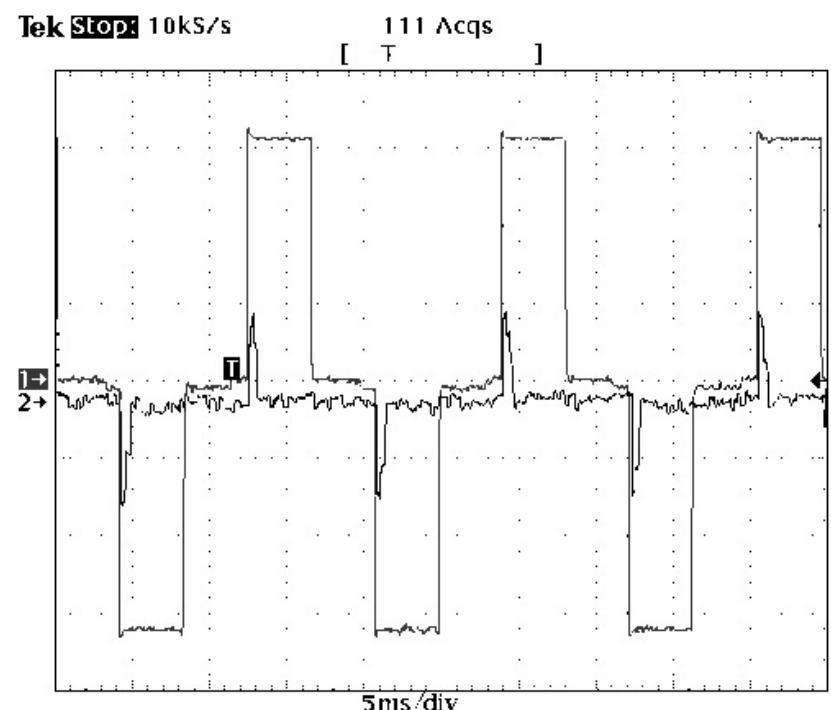

As Figuras 13 e 14 mostram os sinais gerados pelo DSC para o controle geral. Na Figura 13 está a onda triangular para o controle em malha fechada do inversor, com frequência próxima a $120 \mathrm{~Hz}$. A geração da triangular provém de um sinal PWM do DSC56F8006 que por sua vez passa por um filtro RC de três estágios, ligados em cascata, a fim de filtrar as componentes de altas frequências, obtendo assim a envoltória do sinal triangular, através do seu valor médio.

Figura 13 - Sinal PWM do DSC (CH1) e sinal Triangular obtido, após filtragem $(\mathrm{CH} 2)$.

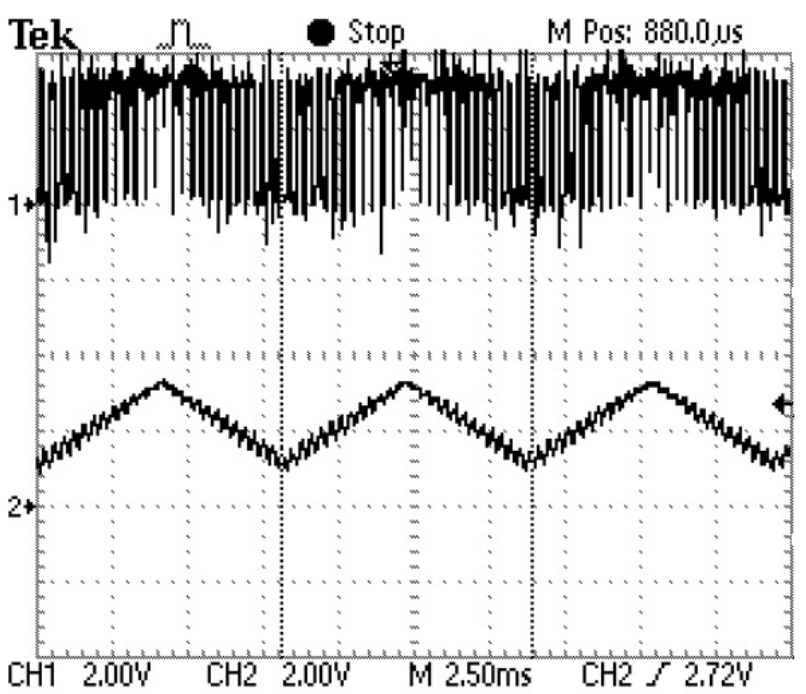

Fonte: Autor

A Figura 14 mostra o pulso de $4 \mu$ s destinado ao controle do inversor e o pulso de 500 ns para sincronizar os blocos constituintes do sistema.

Fonte: Autor 
Figura 14 - Pulsos de controle para o inversor $(\mathrm{CH} 1)$ e de sincronismo $(\mathrm{CH} 2)$ para os blocos constituintes do No-Break.

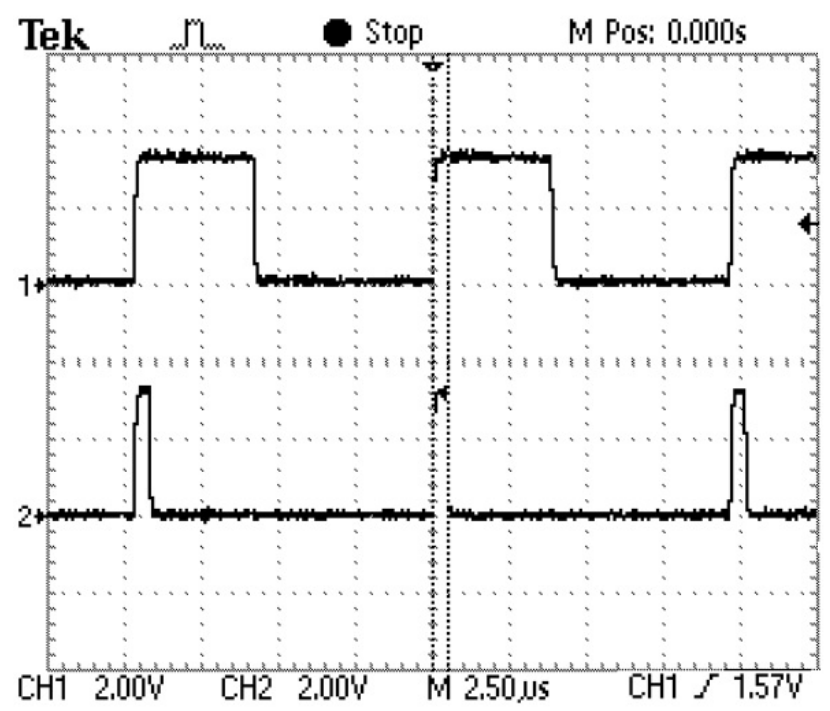

Fonte: Autor

Na Figura 15 está representado o tempo de resposta do DSC (em aproximadamente 650ns) a uma falha de fase na rede elétrica, sendo a onda superior o sinal da rede e a inferior, a resposta do DSC a este evento.

Figura 15 - Detecção da falta de rede (CH1) e pulso de comutação do DSC (CH2).

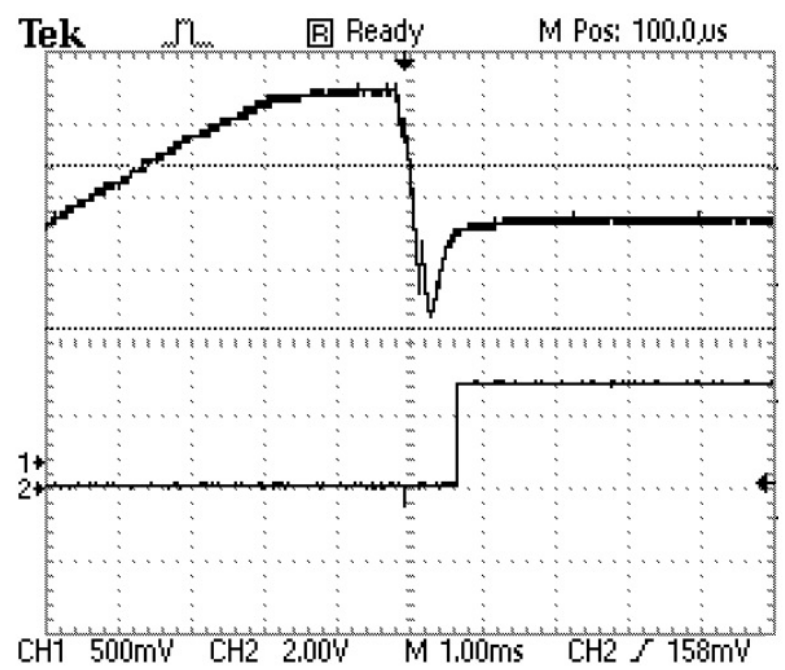

$\mathrm{Na}$ Figura 16 está a comutação entre a rede (senoidal) e o No-Break (forma de onda quasequadrada) assim que ocorre uma falha na rede utilizando cargas lineares na saída do sistema. O tempo de comutação total durou em torno de 7 $\mathrm{ms}$, e portanto, dentro da norma internacional IEC 62040-3/1999 para a classificação dos limites de desvio de onda para comutação entre $1 \mathrm{~ms}$ e $10 \mathrm{~ms}$ (classificação 222).

Figura 16 - Momento da comutação entre a rede e o No-Break.

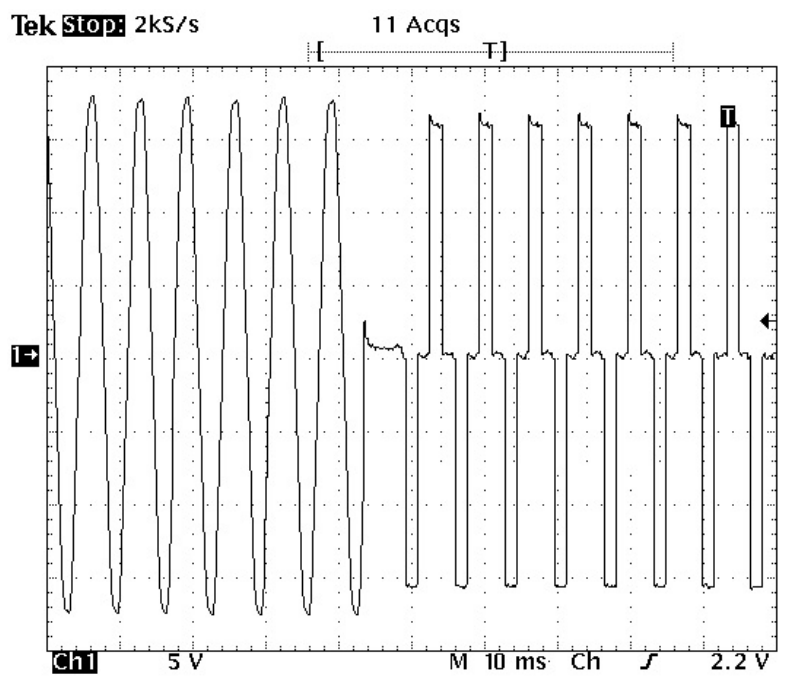

Fonte: Autor

A Figura 17 mostra o protótipo instalado em uma residência.

\section{Fonte: Autor}


Figura 17 - Fotografia do Protótipo do No-Break instalado.

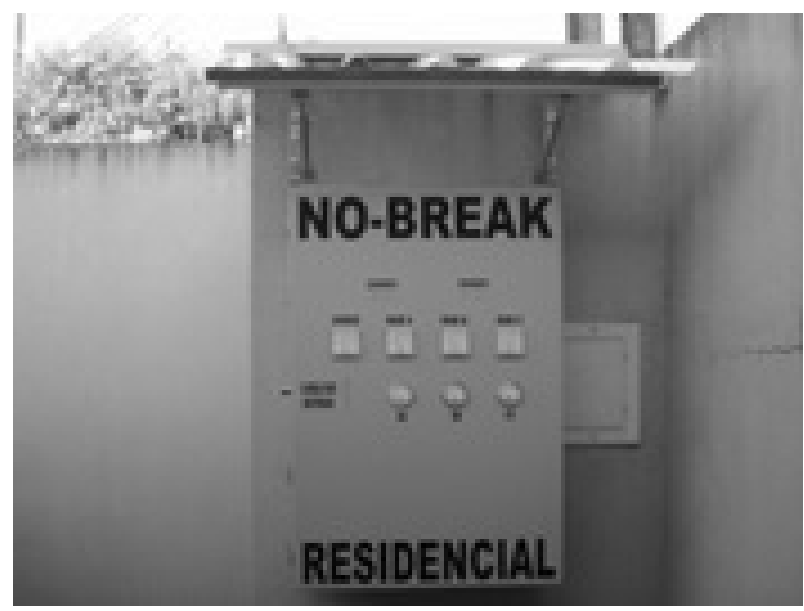

Fonte: Autor

\section{Conclusões}

O protótipo construído tem amplo poder de aplicação. Além da função de No-Break, tem capacidade gerar energia elétrica pela transformação da energia solar em energia elétrica com o uso de painéis fotovoltaicos. Seu funcionamento é regido por diversos fatores, como nível de carga das baterias, acoplamento de painéis solares, luminosidade, entre outros, sendo que todos eles são controlados digitalmente por um sistema central formado por um DSC.

Através dos resultados nos testes realizados em laboratório e em campo e apresentados nesse artigo, foi demonstrado que o protótipo implementado, satisfaz as necessidades de continuidade de energia para residências, comércios e indústrias, devido à capacidade de alimentar cargas resistivas e/ou indutivas e a versatilidade de operar como gerador de energia, com o acoplamento do equipamento a um sistema fotovoltaico.

Quanto maior a potência dos painéis fotovoltaicos e maior for a capacidade de armazenamento do banco de baterias, maior será a autonomia e independência da rede.
Pelos resultados obtidos e a montagem em blocos, este projeto pode ser alterado para qualquer faixa de potência, tornando-o comercialmente interessante.

Todos os circuitos apresentados nos apêndices são fiéis aos montado na prática, tornando-o uma fonte de referência para os diversos setores, sendo assim uma importante contribuição.

\section{Referências}

AHMED, A. Eletrônica de potência. São Paulo: Prentice Hall, 2000.

AHMED, N.A.; LEE, H.W.; NAKAOKA, M. Dual-mode time-sharing sinewave-modulation soft switching boost full-bridge one-stage power conditioner without electrolytic capacitor DC link. IEEE Transactions on Industry Applications, v. 43, n. 3, p. $805-813,2007$.

COSTA, H. S.; Costa, R. A. L. Análise econômica comparativa entre diferentes opções para eletrificação domiciliar rural .Revista Econômica do Nordeste, Fortaleza, v.31, n. 3 p. 320-328, julset. 2000

FREESCALE Tm. MC56F8006 reference manual. 2011. Disponível em: $<\mathrm{http}: / /$ cache.freescale.com/ files/dsp/doc/ref_manual/MC56F8006RM.pdf>. Acesso em: 15 out. 2012.

GILL, A. Introduction to the theory of finite-state machines. New York: McGraw-Hill, 1962.

IBGE. Sinopse do censo demográfico 2010. Disponível em: <http://www.censo2010.ibge.gov. $\mathrm{br} /$ sinopse/index.php?dados $=\mathrm{P} 13 \& \mathrm{uf}=00>$. Acesso em: 15 out. 2012.

SOUZAJUNIOR, J. C.; PAIXÃO, R. R. Controlador digital de sinais: família 56f800/e baseado no mc56f801- microarquitetura e prática. São Paulo: Érica, 2005.

SILVEIRA JUNIOR, C. R. SOUZA, P. H. R. Implementação de controle digital para carregador 
de baterias tipo chumbo-ácido. 2004. Monografia (Graduação) - Universidade Federal de Goiás, Goiânia.

MELLO, L. F. P. Projeto de fontes chaveadas: teoria e prática. São Paulo: Érica, 2011.

MOHAN, N.; UNDELAND, T. M.; ROBBINS, W. P. Power electronics: converters, applications, and design. 3. ed. New York: John Wiley \& Sons, 2003.

RASHID, M. H. Eletrônica de potência: circuitos, dispositivos e aplicações. São Paulo: Makron Books, 1999.

SAMESINA, M. I; REZENDE, J. W.; PEREIRA, G. M. V. Suportabilidade de contatores CA submetidos a afundamentos de tensão (voltage SAGS), Ciência \& Engenharia, v. 15, n. 1, p. 101-106, jan.-dez. 2006.

SOLTER, W. A new international UPS classification by IEC 62040-3. In: INTELEC - Annual International Telecommunications Energy Conference, 24 ${ }^{\text {th }}$, 2002, Québec. Proceedings... Québec: IEEE, 2002. v. 29, p. 541-545.

FRAIDENRAICH, N. Energia solar no Brasil: os próximos 20 anos. Campinas, 2002. Disponivel em: $<$ http://www.feagri.unicamp.br/energia/Ener20/ pdf/caderno.pdf $>$. Acesso em: 15 out. 2012. 


\section{Apêndice 1 - Diagrama de blocos do sistema No-Break.}

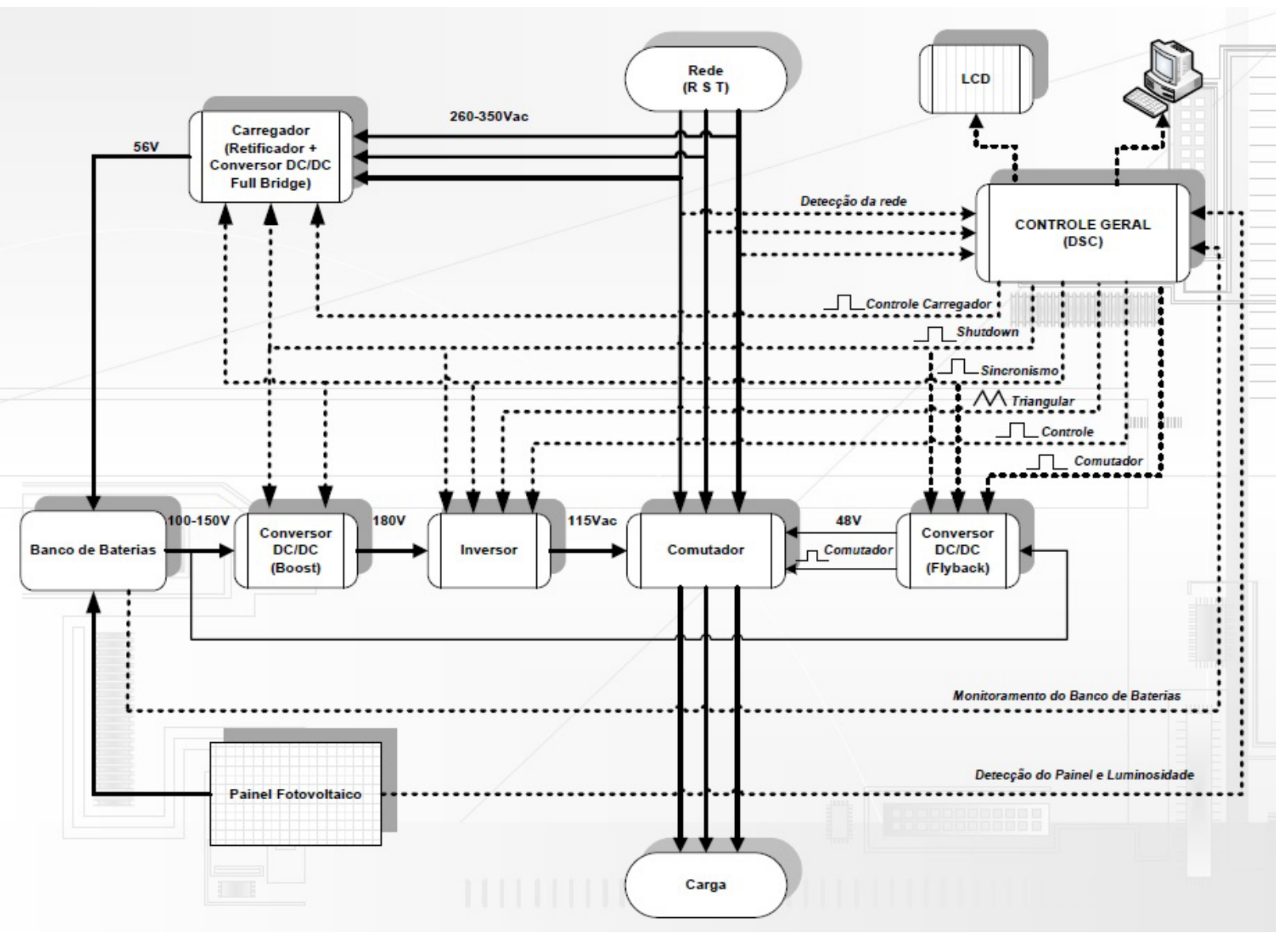




\section{Apêndice 2: Controle DSC.}

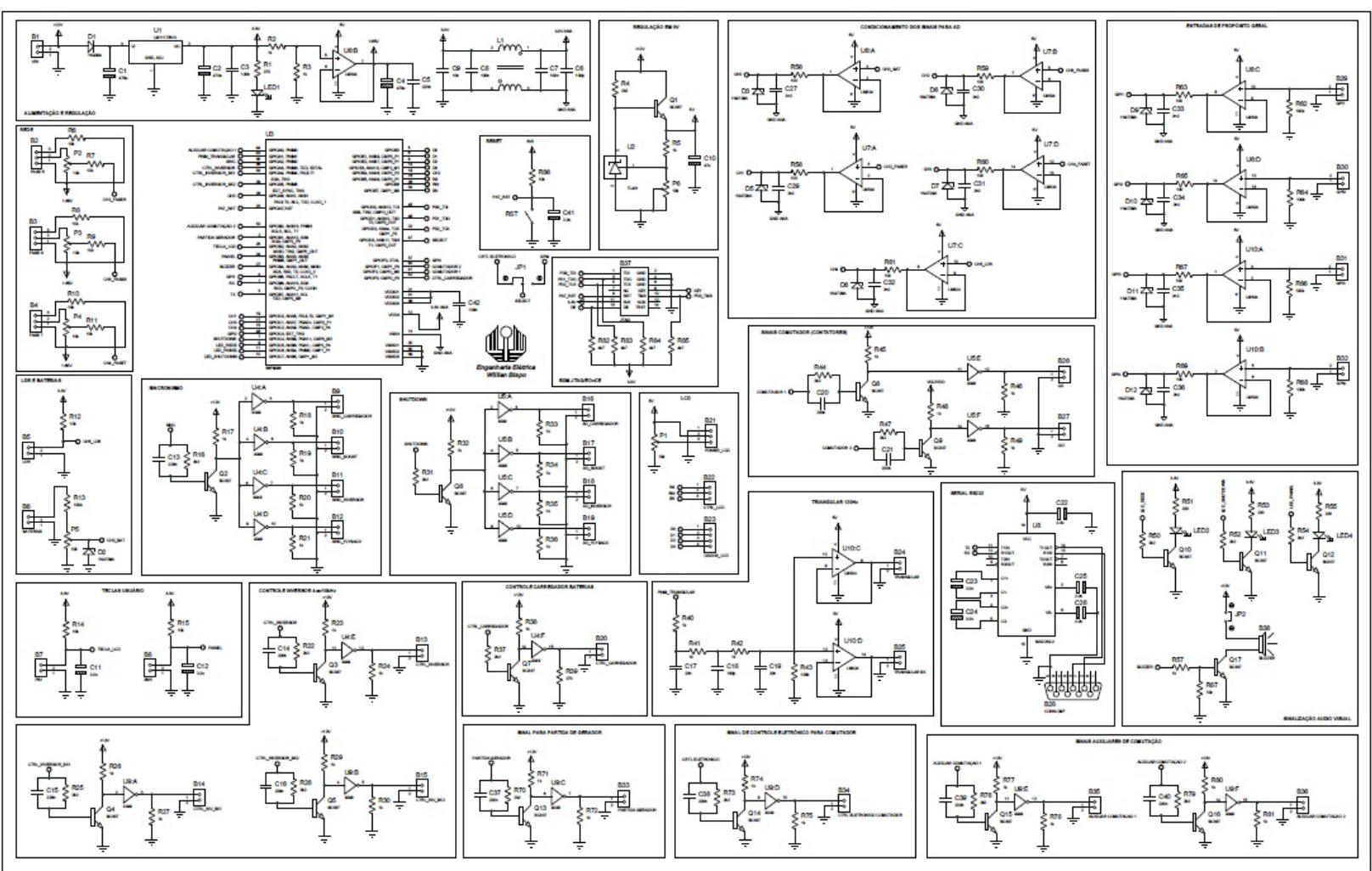




\section{Apêndice 3: Circuito completo Boost.}

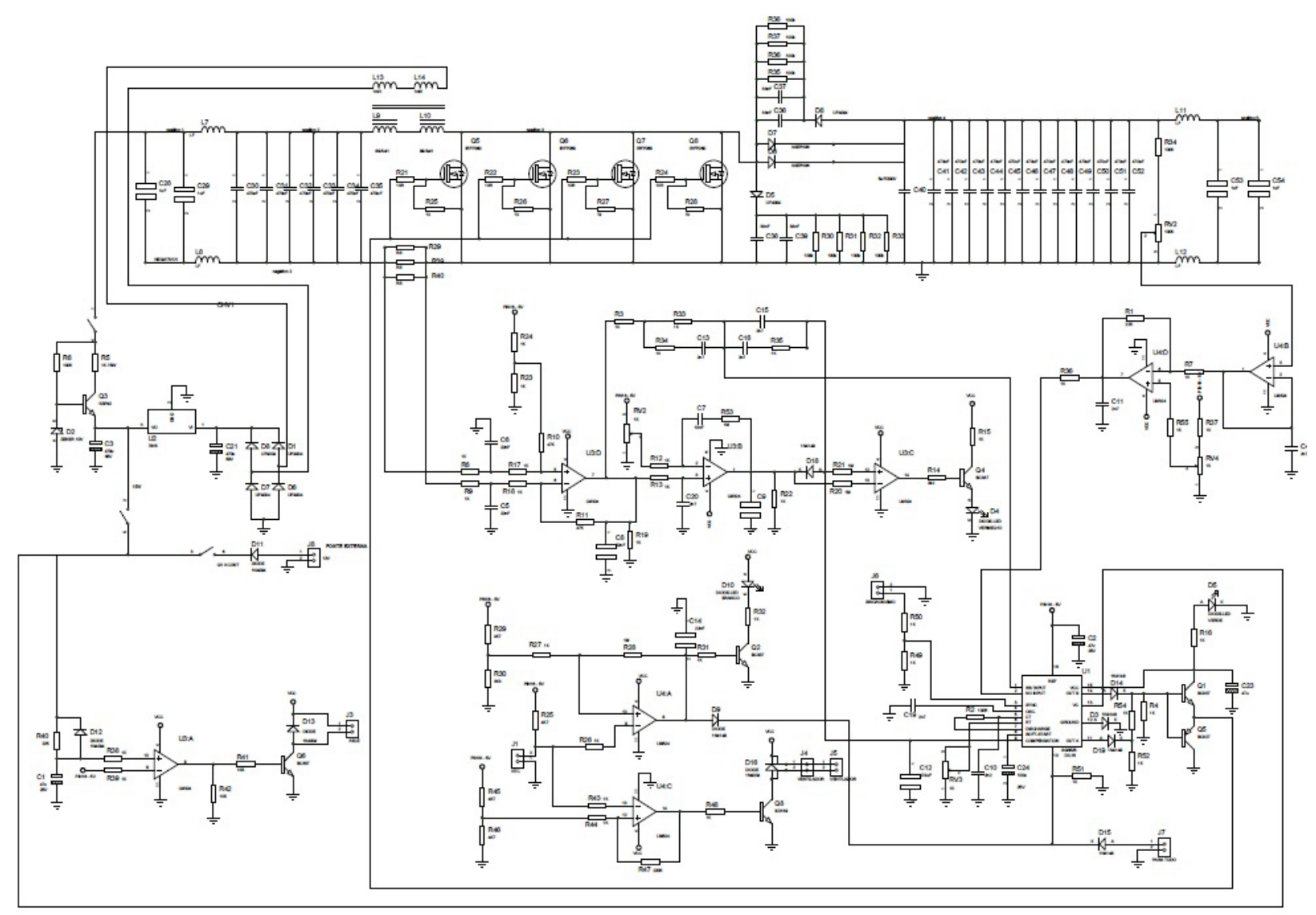

